\title{
Relations of stellar mass and electron temperature-based metallicity of star-forming galaxies in wide mass range
}

\author{
Weibin Shi ${ }^{1,2}$, Yanchun Liang ${ }^{1}$ and Francois Hammer ${ }^{3}$ \\ ${ }^{1}$ Key Laboratory of Optical Astronomy, National Astronomical Observatories, Chinese \\ Academy of Sciences, Beijing 100012, China, email: ycliang@bao.ac.cn \\ ${ }^{2}$ School of Space Science and Physics, Shandong University at Weihai, Weihai 264209, China \\ ${ }^{3}$ GEPI,Observatoire de Paris, Section de Meudon, 92195 Meudon, France
}

\begin{abstract}
We gather a sample of both metal-rich and low metallicity galaxies. Both of them have oxygen abundances estimated from electron temperature (Te). They spread in a wide stellar mass range from $10^{6} M_{\odot}$ to $10^{11} M_{\odot}$. Then, a consistent relation of stellar mass and metallicity are derived from them for such wide range of stellar masses. This relation also shows clearly the discrepancy of Te-based oxygen abundances from those derived from the strong-line method.
\end{abstract}

Keywords. galaxies: abundances, galaxies: evolution, galaxies: statistics

Stellar mass and metallicity are two of the most fundamental physical properties of galaxies to understand the galaxy evolution process. In Fig.1 we present both the metalrich galaxies (red stars, from Liang et al. 2007) and the low metallicity galaxies (blue pluses, from Lee et al. 2006) with oxygen abundances derived from Te. These two samples can be combined well to derive a nice relation of metallicity with stellar mass, which is the black solid line resulted from the least square fit. This is the consistent relation in such wide range from very low to high stellar masses and nicely show a linear relation of the increasing metallicity with increasing stellar mass. Also, this line is much lower than the green solid curve which refers to the SDSS star-forming galaxies from Tremonti et al. (2004) with the strong-line method derived oxygen abundances. This work was supported by the China NSFC Foundation (grant Nos.10933001, 11273026, 11178013 and 11233004) and by the Provincial Natural Science Foundation of ShanDong (ZR2010AM006).

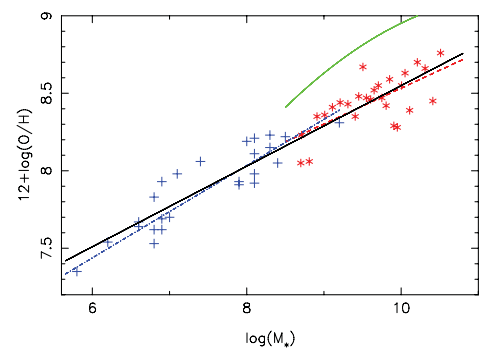

Figure 1. The relations of stellar mass and metallicity from sample galaxies in a very wide stellar mass range (from $10^{6} M_{\odot}$ to $\left.10^{11} M_{\odot}\right)$. The black solid line as $12+\log (\mathrm{O} / \mathrm{H})=5.950+$ $0.260 \times \log M_{*}$ is fitted with the metal-rich (red stars) and low metallicity (blue pluses) ones. The red dashed and blue dot-dashed lines refer to the fits for each of the two samples, respectively.

\section{References}

Lee, H., Skillman, E. D., Cannon, J. M., et al. 2006, ApJ, 647, 970

Liang, Y. C., Hammer, F., Yin, S. Y., Flores, H., et al. 2007, A\&A, 473, 411

Tremonti, C. A., Heckman, T. M., Kauffmann, G., et al. 2004, ApJ, 613, 898 